\title{
Archipel
}

ARCHIPEL Études interdisciplinaires sur le monde insulindien

$97 \mid 2019$

Varia

\section{Bandit Saints of Java : How Java's Eccentric Saints Are Challenging Fundamentalist Islam in Modern Indonesia}

Henri Chambert-Loir

\section{(2) OpenEdition \\ Journals}

\section{Electronic version}

URL: https://journals.openedition.org/archipel/1144

DOI: $10.4000 /$ archipel. 1144

ISSN: 2104-3655

Publisher

Association Archipel

\section{Printed version}

Date of publication: 11 June 2019

Number of pages: $310-312$

ISBN: 978-2-910513-81-8

ISSN: 0044-8613

Electronic reference

Henri Chambert-Loir, "Bandit Saints of Java : How Java's Eccentric Saints Are Challenging

Fundamentalist Islam in Modern Indonesia", Archipel [Online], 97 | 2019, Online since 16 June 2019, connection on 22 September 2021. URL: http://journals.openedition.org/archipel/1144 ; DOI: https:// doi.org/10.4000/archipel.1144 
La troisième et dernière partie est constituée par les traductions de quatre textes plus ou moins contemporains de la chronique, dont certains déjà traduits antérieurement (pp. 205-240) : le bref récit de Cheng Xunwo sur Batavia (sans annotations), un choix de textes se rapportant à Cai Xin 蔡新, un fonctionnaire originaire du Fujian, ayant encouragé Cheng Xunwo à rédiger sa relation de voyage, des extraits du travelogue de Wang Dahai, et une brève note sur Batavia (reproduite dans l'édition de la chronique par Hsü Yün-Ts'iao). Cette partie se termine sur huit appendices (pp. 243-293) donnant la traduction de la nomination du capitaine Tsoa Wanjok en 1678, diverses listes, dont celles des gouverneurs généraux, des chefs de la communauté chinoise et des secrétaires de l'office des Chinois (figurant dans les versions utilisées par Hsü Yün-Ts'iao), ainsi qu'un glossaire des termes malais et néerlandais utilisés dans la chronique.

Les éditeurs ont accompli un travail fort louable. On appréciera tout particulièrement les efforts faits pour éclairer, voire corriger, le texte de la chronique en s'appuyant sur diverses sources hollandaises contemporaines. Les sinologues regretteront toutefois que le manuscrit, qui est à la base de cette traduction, n'ait pas été reproduit. Ils regretteront également que les nombreuses transcriptions de toponymes n'aient pas été données selon la prononciation hokkien, laquelle aurait permis de mieux comprendre les identifications faites par les traducteurs.

Claudine Salmon

George Quinn, Bandit Saints of Java: How Java's Eccentric Saints Are Challenging Fundamentalist Islam in Modern Indonesia, Burough on the Hill, UK: Monsoon Books, 2018, 432 p., ISBN: 9781912049448.

Le culte des saints, c'est-à-dire les pèlerinages effectués sur des tombes musulmanes dites « sacrées », représente, à Java, un aspect relativement négligé de la dévotion religieuse, par opposition à la foi, aux observances et aux rites dictés par le dogme musulman. Il s'agit pourtant d'une pratique courante qui, pour des dizaines de millions de dévôts, réalise la fusion entre la religion, observée par ailleurs, et des croyances beaucoup plus anciennes.

George Quinn consacre à ce phénomène un livre savant, intelligent et subtil, écrit pour le grand public. Il ne s'agit pas de vulgarisation, de mettre un sujet ardu à la portée de lecteurs du commun, mais de traiter un thème des plus sérieux avec simplicité, sur un ton personnel et léger, avec humour aussi, sans s'embarrasser des paraphernalia de l'académisme : très peu de notes et aucune référence bibliographique dans le texte ; l'érudition et les codes académiques sont rassemblés dans une annexe modestement imprimée en petits caractères, marquant une césure entre mode narratif et discours scientifique. Même dans cette section, les références ne sont pas ordonnées dans une classique bibliographie, mais au fil d'un discours sur les sources de l'ouvrage. Cellesci sont en majorité indonésiennes : livres, thèses, articles et blogs. Cela n'exclut en rien l'érudition, bien au contraire. Le livre est nourri d'une connaissance intime de la civilisation javanaise. La maîtrise du javanais a été un atout considérable dans la familiarisation de l'auteur avec les sites. Les textes indonésiens, javanais et arabes cités sont traduits par l'auteur. 
L'ouvrage est composé comme un récit de voyage, le récit d'un promeneur assidu, curieux, toujours bienveillant et respectueux, ouvert à toutes les rencontres et attentif à toutes les informations. La trame du livre est la description de lieux saints d'après l'expérience personnelle qu'en a faite l'auteur durant les trois dernières décennies, ou plus, trame sur laquelle sont tissés des développements sur la légende des saints, l'histoire de l'islam javanais, les traditions javanaises écrites (kakawin, babad, suluk, serat, kitab, wayang) et maints autres aspects de la culture javanaise. Les digressions sont nombreuses, qui nous transportent aux quatre coins du monde javanais, telles celles sur la kiblat (52-56), l'enseignement de Sunan Bonang (69-72), la personnalité de Ph. van Akkeren (79), une représentation de wayang (114-116), l'opium aux Indes Néerlandaises (118-119), l'organisation Pemuda Pancasila (142-148), l'interprétation musulmane du tsunami d'Aceh en 2004 (216-217), le ludruk (243-245), l'histoire de Madura (250-256), la vague d'homophobie publique soulevée en 2015 (261-262), l'histoire de la forêt à Java (321-331), l'affaire Ahok (380-383) et d'autres.

Dans les études sur le culte des saints, comme dans celles sur l'islam indonésien en général, la tendance est au comparatisme, à la synthèse et à la généralisation. Quinn adopte la démarche inverse, celle de l'ethnographie, mais nourrie d'un appareil contextuel de grande ampleur.

Ce parti pris nous prive de réflexions générales sur, par exemple, la personnalité des gardiens de sites (les juru kunci), la sacralisation de l'espace, ou encore le rapports aux défunts.

Quinn ne tente pas une définition de la sainteté, ni même de lieu sacré. Il décrit des sites où, d'une manière générale, sont vénérés des personnages plus ou moins historiques, plus ou moins authentiques. Beaucoup de sites sacrés, à Java, sont en marge d'une définition, même aussi rudimentaire : le chapitre sur le gardien rituel du Merapi (Mbah Maridjan) et le rite du Labuhan (p. 213-242) ne concerne pas une tombe ni un saint - bien qu'elle aboutisse à la naissance d'un saint, via la vénération d'une tombe incertaine ; le vestige vénéré sur le site de Nyai Ageng Bagelen (23-34) n'est pas une tombe musulmane, mais un stupa, tandis que d'autres sections sont consacrées à un arbre (286-293), une grotte (Gua Langse, 295), ou encore un gong, tous sacrés (332).

Le livre, déjà volumineux, est loin d'être exhaustif : il y manque des sites importants, tels par exemple que Trusmi, Kemukus, Imogiri, Kota Gede, ou Malik Ibrahim. Mais ces absences, que certains regarderont peut-être comme des lacunes, sont délibérées et parfaitement maîtrisées.

Le narrateur voyage : il traverse Java d'une côte à l'autre et d'une extrémité à l'autre, de même que certains pèlerins javanais circulent de tombe en tombe, afin d'accumuler les mérites, les pouvoirs spirituels et les enseignements. Et le lecteur progresse ainsi, d'une tombe, d'une légende, d'une leçon d'histoire à une autre, accumulant les images et les informations sur la religion et la culture javanaise. Livre initiatique, en somme, sous forme de promenade, constamment passionnante et renouvelée, mais qui ne peut avoir de fin, car le sujet est infini.

Le parti provocateur du titre n'est absolument pas dans le ton du livre, qui est au contraire constamment mesuré et subtil. Il existe des saints-bandits repentis, excentriques, voire hérétiques ; ils sont représentés ici par Sunan Kalijaga, Maling Gentiri, Ki Ageng Balak, Ki Boncolono, et encore Seh Siti Jenar et Sunan Panggung; mais ils sont loin d'être majoritaires. Quant au conflit entre le culte des saints et l'islam fondamentaliste, qui est une constante de l'islam indonésien depuis le 15e 
siècle, il est sous-jacent dans nombre de chapitres et explicitement discuté à propos de Mbah Priok (181-212) et de Sunan Bayat (367-372). Ce dernier chapitre débouche sur une longue comparaison (372-409) entre les dévotions accomplies sur le site-même et dans la mosquée adjacente, avec la conclusion, solidement argumentée, que le pôle abangan-kramat (l'islam javanisant), majoritaire au lendemain de l'indépendance, est devenu aujourd'hui minoritaire, au profit du pôle santri-masjid (l'islam rigoureux ou tout bonnement orthodoxe). Parmi les nombreuses causes de ce renversement (urbanisation, éducation, internationalisation...), la tragédie de 1965 eut le rôle d'un catalyseur brutal. Avec la paradoxale constatation, cependant, que les pèlerinages sur les tombes n'ont cessé d'augmenter de façon spectaculaire depuis les années 1980 jusqu'à aujourd'hui, précisément par réaction envers la vague d'orthodoxie coercitive. Sur le plan religieux, les lieux saints (kramat) sont le refuge de la culture abangan (permissive), mais les tombes, plus ou moins habillées de signes d'orthodoxie ou de « santrification » (402), sont aussi visitées par des orthodoxes.

Au terme de ce cheminement ethnographique et savant à la fois, le lecteur découvre une forme de dévotion et de foi musulmane à l'opposé de tout ce que lui enseigne l'actualité internationale dont rendent compte les médias et même très éloignée de l'islam dont parlent les publications académiques sur l'Indonésie. La vénération des tombes, ainsi que d'autres sites sacrés, est indubitablement musulmane, mais elle s'inscrit dans un univers spirituel, qui représente un aspect essentiel du rapport des Javanais à l'au-delà. En filigrane à la visite des tombes, cet ouvrage présente une exploration de la relation historique, géographique et spirituelle, à Java, entre les mondes visible et invisible et entre le microcosme et le macrocosme.

Henri Chambert-Loir

Urip Iku Urub: Untaian Persembahan 70 Tahun Profesor Peter Carey ("La Vie comme un flambeau : Un collier d'offrandes pour les 70 ans du Professeur Peter Carey »), Jakarta : Kompas, 2019, xc-568 p., 120 illustrations, index. ISBN: 978-602412-534-9.

Comme souvent dans les hommages offerts à des professeurs d'université, un corps d'articles, dans ce volume, est constitué de souvenirs et de documents personnels, et un autre d'essais en rapport avec la discipline du professeur en question.

La première partie (c.130 p.) est consacrée à Peter Carey, à commencer par un texte autobiographique de celui-ci (illustré par 55 photographies éparses dans la première partie de l'ouvrage), qui fait une large place à ses origines et à son enfance.

Né en 1948 à Rangoon, où il passe son enfance, Peter Carey fait ses études en Angleterre, puis des études supérieures à Cornell et à Leyde; il fait durant deux ans des recherches à Java et devient professeur d'histoire de l'Europe moderne à Oxford, où il enseignera pendant 30 ans. Il passe sa thèse sur Diponegoro en 1977, mais elle ne sera publiée que trente ans plus tard parce que son parcours suit des détours imprévus. Il consacre, en effet, une grande partie de son temps, dans les années 19801990, à une campagne de soutien au Timor oriental, lors de la difficile transition qui 